\title{
Coexisting and possible primary extra-gastrointestinal stromal tumors of the pancreas and liver: A single case report
}

\author{
LEI LIU ${ }^{1}$, YINGQIAO ZHU ${ }^{1}$, DONGXUAN WANG ${ }^{1}$, \\ CHANGBIN YANG ${ }^{2}$, QI ZHANG ${ }^{3}$, XIUKUN LI $^{4}$ and YANG BAI ${ }^{1}$ \\ ${ }^{1}$ Department of Ultrasound, First Hospital of Jilin University, Changchun, Jilin 130021; ${ }^{2}$ Department of Radiation Oncology, \\ The Tumor Hospital of Jilin Province; ${ }^{3}$ Department of Ultrasound, College of Pharmacy, Jilin University, Changchun, \\ Jilin 130012; ${ }^{4}$ Ultrasound Department, Jilin Provincial People's Hospital, Changchun, Jilin 130021, P.R. China
}

Received December 11, 2014; Accepted January 18, 2016

DOI: $10.3892 / \mathrm{ol} .2016 .4420$

\begin{abstract}
Gastrointestinal stromal tumors (GIST) are mesenchymal neoplasms of the gastrointestinal tract (GI) that are defined, in part, by the expression of CD117, a c-Kit proto-oncogene protein. GISTs emerge outside of the GI at a very low frequency, typically in a single organ or location. GISTs that occasionally emerge outside of the GI are classified as extra-gastrointestinal stromal tumors (EGIST). The present study reports an extremely rare case of EGIST detected in the pancreas and the liver. The pancreatic and liver tumors were $4.5 \times 2.5 \mathrm{~cm}$ and $2.0 \times 1.5 \mathrm{~cm}$ in size, respectively. Both tumors consisted of CD117-positive spindle cells with a similar mitotic rate of 1-2 per 50 high power fields. The pancreatic and the hepatic EGISTs were at a low risk of malignancy, and both tumors were proposed to be primary stromal tumors. To the best of our knowledge, this is the first report of likely primary EGIST identified in the pancreas and liver of the same patient.
\end{abstract}

\section{Introduction}

Gastrointestinal stromal tumors (GIST) are mesenchymal neoplasms of the gastrointestinal (GI) tract, the incidence of which is low $10-20$ per 100,000 individuals in China $(1,2)$. The mainstay of treatment is resection, and anticancer and biological therapy may be used in cases of extensive metastasis. One of the characteristic features of GISTs is the expression of CD117, a c-Kit proto-oncogene protein. The tumors most commonly occur in the GI tract from the esophagus to the anus (3-5); however, tumors expressing CD117 occasionally arise in locations adjacent to, but outside of, the GI tract. GISTs have been

Correspondence to: Dr Yingqiao Zhu, Department of Ultrasound, The First Hospital of Jilin University, 71 Xinmin Street, Changchun, Jilin 130021, P.R. China

E-mail: zhuyingqiaoZYQ@163.com

Key words: gastrointestinal stromal tumors, extra-gastrointestinal stromal tumors, primary tumor, metastasis, CD117, interstitial cells of Cajal reported in the omentum, mesentery, gallbladder, prostate and retroperitoneum (6-9). GISTs arising outside of the GI track are classified as extra-gastrointestinal stromal tumors (EGISTs) (9). EGIST is rare, with an estimated incidence of 1.5-6.0\% worldwide $(1,2)$. However, due to the rarity of EGIST, mortality rates remain unclear. EGIST refers to tumors derived from the omentum, mesentery or retroperitoneal soft tissue; however, these tumors originate in the stroma and possess no definitive type of cell differentiation, and are not associated with intestinal and digestive tract serosa (7). EGIST has been reported in the omentum, mesentery, bladder, gallbladder, pancreas, uterus and retroperitoneum $(1,6,7,10)$. The treatment for EGISTs is resection, if possible. Primary pancreatic EGISTs are rare in the clinic and in the literature, with only $\sim 20$ cases reported to date (11-28). Similarly, hepatic EGIST is also rare (29-31). The occurrence of a primary pancreatic EGIST accompanied by a primary hepatic EGIST in the same patient is, therefore, considered to be an extremely rare event. The present study reports a case of primary pancreatic EGIST accompanied by an apparent primary hepatic EGIST.

\section{Case report}

A 56-year-old female patient was admitted to The First Hospital of Jilin University (Changchun, China) in July 2014, 10 days after solid lesions were detected in the pancreas and liver during a routine physical examination at the same hospital. Findings from general examination upon admission included: No skin or sclera jaundice, abdominal pain, bloating, nausea, vomiting, discomfort or palpable abdominal mass. There was also no history of pancreatitis or abdominal trauma. A $5.2 \times 3-\mathrm{cm}$ hypoechoic lesion was detected in the pancreatic body and a $2.4 \times 1.7-\mathrm{cm}$ hypoechoic lesion was detected in the left hepatic lobe by ultrasound (iU Elite; Philips Healthcare, Andover, MA,USA) Fig. 1A and B, respectively), indicating the existence of space-occupying lesions in the pancreas and liver. To further characterize the lesions following ultrasound, the patient underwent analysis with contrast-enhanced ultrasonography (CEUS), using SonoVue ultrasound contrast agent (Bracco, Milan, Italy) and color Doppler diagnostic apparatus (model LOGIQ E9; GE Healthcare, Pittsburg, PA, USA). The lesion at the pancreatic body was highly enhanced in the 
A

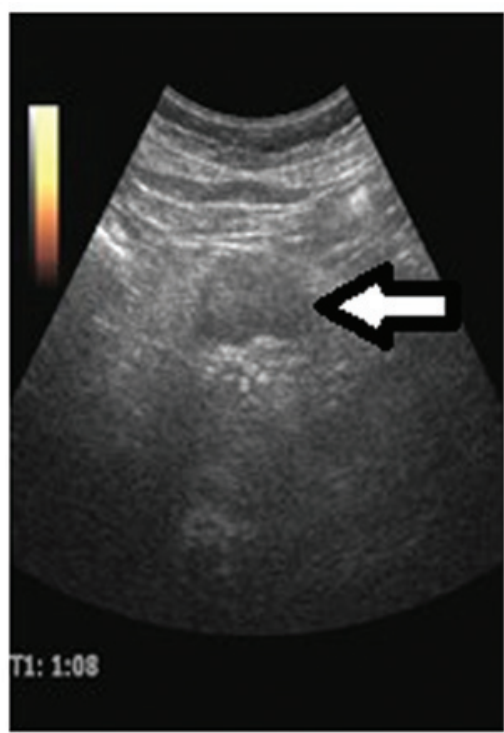

B

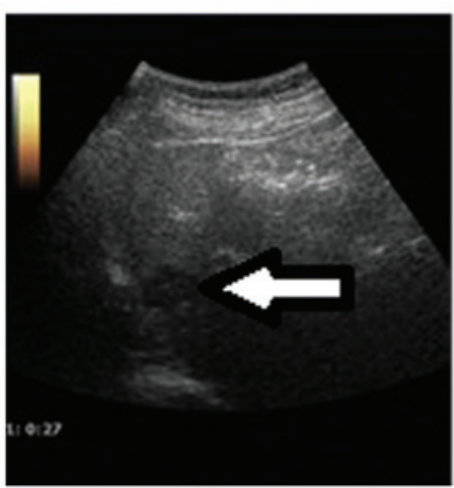

Figure 1. Ultrasound images revealing hypoechoic lesions (arrows) at the (A) pancreatic body and (B) hepatic left lateral lobe.

arterial phase by CEUS and a washout of this enhancement was observed in the venous phase (Fig. 2A). The lesion at the left lobe of liver was quickly and highly enhanced at the periphery of the lesion (rim enhancement) in the arterial phase, but it began to fade in the venous phase (Fig. 2B). The washout of the enhancement in the liver lesion occurred considerably earlier than in the surrounding hepatic parenchyma. The CEUS results suggested that the lesion at the pancreatic body was a primary malignant tumor, while the lesion in the left hepatic lobe was likely a metastatic malignant tumor. Plain and enhanced computed tomography (CT) scans were subsequently performed by intravenous injection with the contrast agent iopromide (Bayer Healthcare, Whippany, NJ, USA) and scanning with a dual-source 64-slice SOMATOM Definition AS (Siemens AG, Berlin, Germany). Plain and enhanced CT scanning detected a $5.7 \times 2.7-\mathrm{cm}$ visible low-density lesion in the pancreatic body, and the image was intensified relatively poorly on the enhanced scan (Fig. 3). The plain CT also revealed a $2.2-\mathrm{cm}$ diameter low-density lesion close to the top of the left hepatic lobe near the diaphragm, and a slight edge enhancement was observed on the enhanced scan (Fig. 4). The CT scan results suggested a pancreatic cancer accompanied by a low-density lesion in the liver, and could not rule out the possibility of hepatic metastasis. The clinical diagnosis, therefore, was malignant pancreatic cancer with hepatic metastases.
A

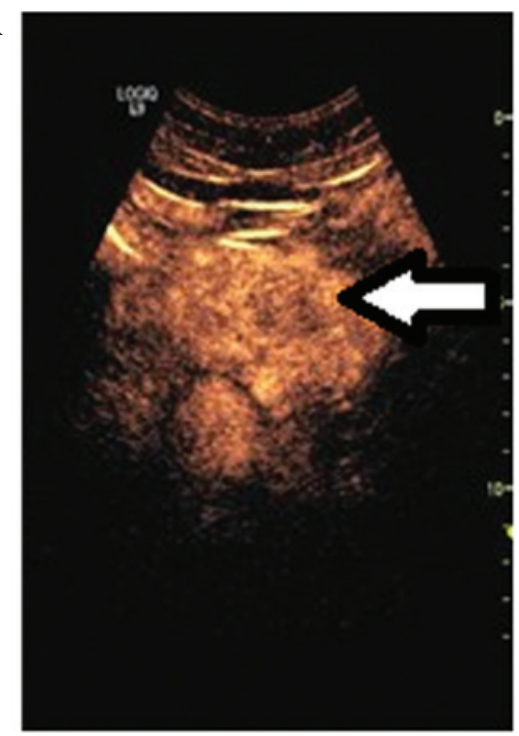

B

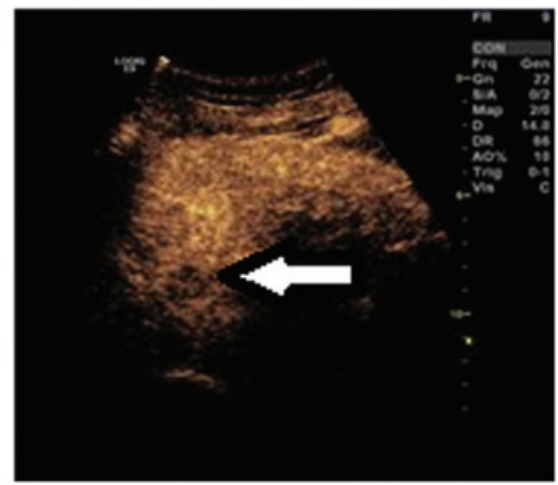

Figure 2. Contrast-enhanced ultrasonography images following injection with Sonovue ultrasound contrast agent revealing (A) high-contrast enhancement (arrow) in the arterial phase at the pancreatic body and (B) quick rim enhancement (arrow) in the arterial phase at the periphery of the hepatic left lateral lobe lesion.

Exploratory laparotomy was subsequently performed. Tumors measuring $\sim 4.5 \times 2.5 \times 2.0 \mathrm{~cm}$ and $\sim 2.0 \times 1.5 \times 1.5 \mathrm{~cm}$ were identified in the pancreatic body and the left hepatic lateral lobe, respectively, during the probing procedure. Lesion cells were collected by fine needle aspiration and cytological examination revealed atypical cells, with an increased cell volume and enlarged and mitotic nuclei, suggesting the possibility of malignant pancreatic and hepatic tumors. Following receipt of written informed consent from the patient's family, tumor biopsy procedures for pathological diagnosis were conducted. Biopsy tissues were fixed in $10 \%$ neutral buffered formalin (Fuzhou Maixin Biotech Co., Ltd., Fuzhou, China), embedded in paraffin (Leica Microsystems, Wetzlar, Germany) and sectioned (3-5 $\mu \mathrm{m}$ thickness; model no., 2235; Leica Microsystems). The sections were stained with hematoxylin and eosin (Fuzhou Maixin Biotech Co., Ltd.) and observed under a microscope (Olympus BX51; Olympus Corporation, Tokyo, Japan). Pathological findings in the hepatic and pancreatic biopsy tissues indicated that the tumors were mitotic spindle cell tumors with mitotic activity of $1-2$ per 50 high-power fields (HPF; Fig. 5). The stromal tumor diagnosis was further supported by the following immunohistochemical staining results in liver and pancreas tissues: Vimentin (+), CD117 (+), 
discovered on GIST-1 (+), Ki-67 (60\% +), S-100 (-), CD34 (-), cytokeratin (-), smooth muscle actin (-), Des (-) and epithelial membrane antigen (-). Each tumor was treated separately. On July 9, 2014, $42{ }^{125}$ Iodine radioactive particles (half life, 2 months; energy/particle, $28.37 \mathrm{keV}$; Shanghai Xinke Pharmaceutical Co., Ltd., Shanghai, China) were implanted in the pancreatic cancer lesion and a microwave ablation therapy was used to target the hepatic lesion. Following these therapeutic techniques, on July 13, 2014, oral Gleevec (400 mg; Novartis, Basel, Switzerland) was administered daily for the following 3 years. The combination of these adjuvant therapies is a novel strategy. The patient is currently undergoing close follow-up via telephone interview to determine the efficacy of this novel treatment strategy, and at each follow-up is advised to continue taking Gleevec. Follow-up telephone interviews were conducted in October 2014, January 2015 and June 2015. The most recent follow-up was in December 2015, and the patient reported that she had presented at another hosptial with abdominal metastasis. Currently, the patient is alive. Written informed consent was obtained from the patient for the publication of the present study.

\section{Discussion}

A conclusive diagnosis of EGISTs relies on pathological examination of the tumor specimen (4). The finding in the present study that both tumor tissues consisted of spindle cells provided direct evidence for the diagnosis of stromal tumors in the pancreas and the liver. Additional evidence was obtained from immunophenotyping of tumor cells by immunohistochemical staining, which revealed positive CD117 expression in the tumor cells. CD117, a c-Kit receptor tyrosine kinase, is the fingerprint marker that differentiates GISTs from true smooth muscle tumors and it is expressed in 95\% of GISTs (12,32-34). Thus, the final diagnosis in the present case of two extra-GI tumors of the pancreas and liver is reliable and valid. However, the next challenge was determining whether the hepatic EGIST was primary or metastatic. Accurate differentiation of in situ tumors from metastatic tumors is important during the selection of treatment strategies, prediction of prognosis and provision of psychological patient care, as the actions taken in all these cases fundamentally depend on the extent of tumor malignancy. When two different sizes of stromal tumors were identified in the pancreas and liver, there were two possibilities: i) Both tumors were primary or ii) one tumor was metastatic. If one of the tumors was not primary, the likelihood of liver metastasis was high, as the hepatic tumor was half the size of the pancreatic tumor. If the hepatic EGIST was considered metastatic, the pancreatic EGIST had to be highly malignant. When all data was re-evaluated during the writing of the current report, uncertainty regarding the malignancy of the pancreatic tumor was raised.

The following are evidence and reasoning supported a diagnosis of primary hepatic EGIST in the present study: i) The most widely accepted criteria used to predict stromal tumor behavior are tumor size and mitotic rate (4), and the pancreatic tumor size $(4.5 \times 2.5 \times 2.0 \mathrm{~cm})$ and mitotic activity $(1-2 / 50 \mathrm{HPF})$ in the current case suggested a low risk of malignancy. Thus, the low risk of malignancy indicated that the probability of metastasis was also low. ii) Furthermore, considering the low

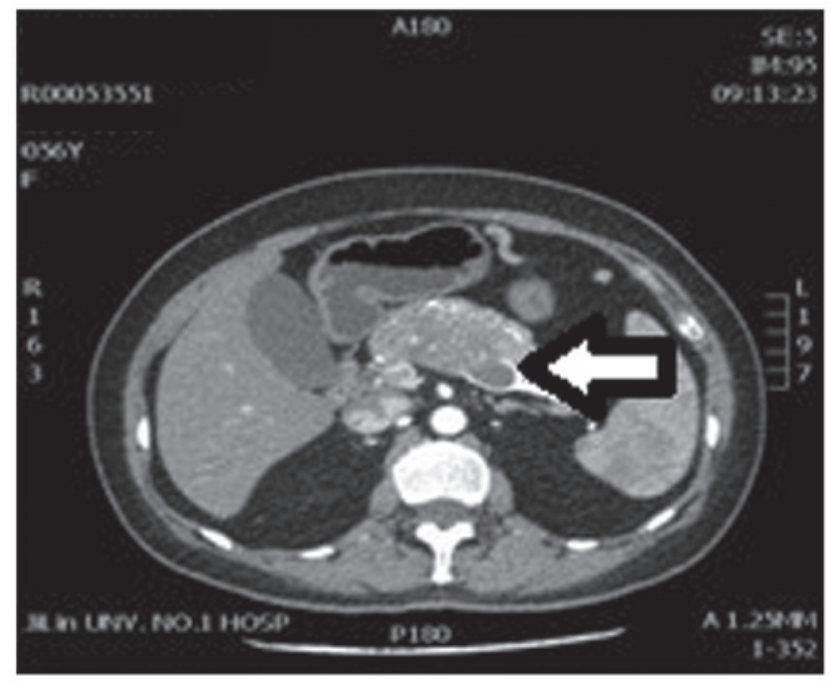

Figure 3. Computed tomography contrast-enhanced image revealing a relatively low degree of enhancement at the pancreatic body lesion (arrow) following intravenous injection with the contrast agent iopromide.

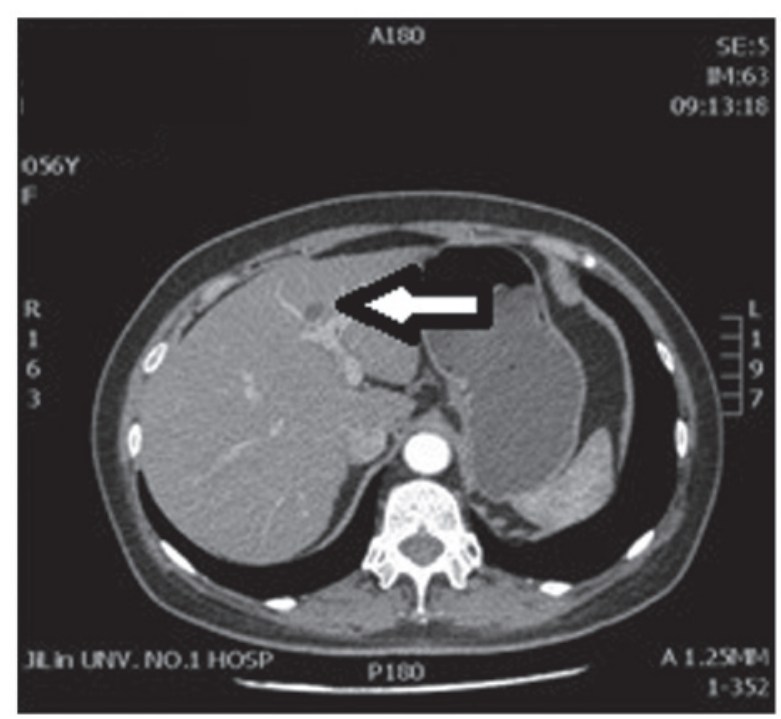

Figure 4. Computed tomography contrast-enhanced image revealing slight enhancement at the periphery of the lesion of the left hepatic lateral lobe (arrow) following intravenous injection with the contrast agent iopromide.

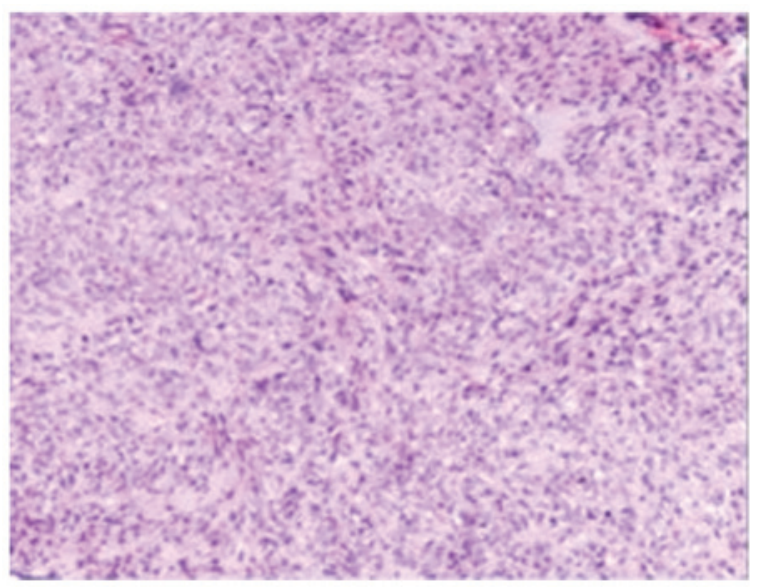

Figure 5. Hematoxylin and eosin staining of the pancreatic biopsy specimen revealing a stromal tumor composed of spindle cells (magnification, x100). 
risk of malignancy, it is unlikely that the pancreatic tumor began metastasizing when the tumor was $2.5 \mathrm{~cm}$, which is the estimated size of the tumor assuming that the primary and metastatic tumors are growing at a similar rate. For example, in a previous patient with a pancreatic EGIST of $6 \times 5 \mathrm{~cm}$ and 12-15 mitoses per $50 \mathrm{HPF}$, no metastasis in the form of a liver space-occupying lesion was observed until 2 years later (25). iii) Finally, there was a possibility that a mural GIST experienced extensive extramural growth, resulting in an eventual loss of connection with the gut wall (9). The lost tumor cells may have first successfully seeded in the pancreas, before later seeding in the liver, as primary EGIST of liver has been previously reported (29-31).

An important consideration is that when a non-stromal tumor is detected in the liver subsequent to the detection of a malignant tumor in other organs, such as the colon/rectum, lung or breast, it can generally be safely assumed that the tumor detected in the liver is metastatic. However, two possibilities exist when the same stromal tumor cell types are identified at simultaneously in two different locations: i) The first possibility is that both tumors are primary and ii) the second is that one of the tumors is metastatic. If the tumor is at a low risk of malignancy, the hepatic EGIST may have been deposited from the same origin that seeded the pancreatic EGIST; however, if the malignancy of the tumor is high, the hepatic tumor may have originated from the pancreatic EGIST. The existence of these two possibilities highlights the challenge of differentiating between primary and metastatic EGIST. If the two EGISTs were simultaneously identified in two different organs and if both EGISTs were at low risk of malignancy then the possibility of a primary tumor must be considered.

CEUS demonstrated rim enhancement at the periphery of the lesion in the left lobe of the liver in the arterial phase that began to fade in the venous phase. The washout of the enhancement occurred considerably earlier in the lesion than in the surrounding hepatic parenchyma. However, these findings only suggest the presence of a likely hepatic tumor and, furthermore, could not distinguish between a primary and a metastatic tumor, as the two tumor types may exhibit similar rim enhancements at their periphery by CEUS $(35,36)$.

CEUS has been increasingly used for the early diagnosis of metastatic tumors in the liver following the confirmation of primary malignant cancer in the colon/rectum, lung, GI tract, pancreas or breast, as malignant tumors in those organs are associated with a high frequency of liver metastasis. The typical CEUS findings in nearly all hypovascular metastasis include varying degrees of contrast enhancement in the arterial phase, particularly in the periphery (rim enhancement) (35). However, an irregular rim enhancement in the periphery of the lesion is also frequently observed in primary liver cancer (36); therefore, this feature is not a specific enough to distinguish between a metastatic and primary lesion. Thus, CEUS findings can only serve as an indicator of the presence or absence of a space-occupying lesion in the liver. The suggestion of metastasis by CEUS is predominantly based on the presence of malignant tumors previously confirmed in other organs. Therefore, as established in the current case, the simultaneous identification of low malignancy stromal tumors in the pancreas and the liver make it impossible for CEUS findings alone to provide definitive evidence of metastasis.
The origin of EGISTs remains controversial, however, at least two hypotheses appear reasonable. One hypothesis assumes that interstitial cells of Cajal (ICCs) are the likely source of the genesis of GISTs (7). ICCs are pacemaker cells that are located throughout the wall of the GI tract, as their function is to regulate the movement of the GI track. If ICCs are the origin cells of EGISTs, the extensive presence of ICCs along the GI track presents an anatomical and pathophysiological opportunity for the occurrence of GISTs in any part of GI track. Additionally, the distribution of ICCs also enables a scenario in which ICC-derived tumor cells may escape and deposit outside of the GI track. For example, the extensive extramural growth of mural GISTs may result in complete loss of contact with the muscularis propria (9), leading to tumor cells being scattered outside of the GI tract. Not all tumor cells deposited in different organs or locations would survive; however, the survival, growth and proliferation of just one or more cells may result in the generation of one or more primary EGISTs in different organs or locations (37). This theory provides a reasonable explanation for the emergence of primary EGIST in the pancreas and liver in the present study. The molecular fingerprint of ICC is the expression of the c-Kit receptor tyrosine kinase (CD117 antigen). The positive detection of CD117 in the pancreatic and hepatic EGIST cells in the current case is in agreement with this hypothesis. The alternate hypothesis is that GISTs actually arise from a common precursor cell of ICCs and smooth muscle cells, which accounts for their ability to grow inside and outside of the GI tract (37). Molecular investigations have confirmed the presence of Cajal-like interstitial cells within extra-digestive organs and vessels. For example, the existence of ICCs has recently been demonstrated in the human exocrine pancreas, and these cells have a phenotype similar to that of enteric ICCs (38). This theory indicates that primary EGISTs may simultaneously occur in different organs of the same patient when the conditions for facilitating the growth of Cajal-like cells are triggered in these locations. Although each hypothesis postulates a different origin, both describe a scenario where it is possible for more than one primary EGIST to simultaneously emerge in different organs or locations.

Surgery represents the first choice for treating resectable pancreatic and hepatic EGISTs (33). For unresectable patients, the tyrosine kinase inhibitor imatinib mesylate (Gleevec) is the primary drug available for treatment (39). In the present case, although unable to perform a complete surgical resection, radioactive particles were implanted into the pancreatic tumor and a microwave therapy technique to target the hepatic EGIST was performed. Following these therapeutic techniques, oral Gleevec was regularly administered. The combination of these adjuvant therapies is a novel strategy. The patient is currently undergoing close follow-up to determine the efficacy of this novel treatment strategy, and at each follow-up appointment is advised to continue taking Gleevec. The most recent follow-up appointment was in November 2015, and the patient presented with abdominal metastasis.

In summary, the present study reports a case with EGIST diagnosed in the pancreas and liver. To the best of our knowledge, this report is the first to provide evidence that primary EGIST can emerge independently in two different organs of the same patient. The present study highlights the 
underappreciated clinical challenge of differentiating between primary and metastatic stromal tumors when both tumors have a low malignancy risk and are detected simultaneously. The possible mechanism by which two primary EGISTs may arise in two different organs is also discussed.

\section{References}

1. Joensuu H, Fletcher C, Dimitrijevic S, Silberman S, Roberts $P$ and Demetri G: Management of malignant gastrointestinal stromal tumours. Lancet Oncol 3: 655-664, 2002.

2. Agaimy A and Wünsch PH: Gastrointestinal stromal tumours: A regular origin in the muscularis propria, but an extremely diverse gross presentation. A review of 200 cases to critically re-evaluate the concept of so-called extra-gastrointestinal stromal tumours. Langenbecks Arch Surg 391: 322-329, 2006.

3. Hirota S, Isozaki K, Moriyama Y, Hashimoto K, Nishida T, Ishiguro S, Kawano K, Hanada M, Kurata A, Takeda M, et al Gain-of-function mutations of c-kit in human gastrointestinal stromal tumors. Science 279: 577-580, 1998.

4. Sarlomo-Rikala M, Kovatich AJ, Barusevicius A and Miettinen M: CD117: A sensitive marker for gastrointestinal stromal tumors that is more specific than CD34. Mod Pathol 11: 728-734, 1998

5. Joensuu H, Roberts PJ, Sarlomo-Rikala M, Andersson LC, Tervahartiala P, Tuveson D, Silberman S, Capdeville R, Dimitrijevic S, Druker B and Demetri GD: Effect of the tyrosine kinase inhibitor STI571 in a patient with a metastatic gastrointestinal stromal tumor. N Engl J Med 344: 1052-1056, 2001.

6. Fletcher CD, Berman JJ, Corless C, Gorstein F, Lasota J, Longley BJ, Miettinen M, O'Leary TJ, Remotti H, Rubin BP, et al: Diagnosis of gastrointestinal stromal tumors: A consensus approach. Hum Pathol 33: 459-465, 2002.

7. Miettinen M, Monihan JM, Sarlomo-Rikala M, Kovatich AJ, Carr NJ, Emory TS and Sobin LH: Gastrointestinal stromal tumors/smooth muscle tumors (GISTs) primary in the omentum and mesentery: Clinicopathologic and immunohistochemical study of 26 cases. Am J Surg Pathol 23: 1109-1118, 1999.

8. Reith JD, Goldblum JR, Lyles RH and Weiss SW: Extragastrointestinal (soft tissue) stromal tumors: An analysis of 48 cases with emphasis on histologic predictors of outcome. Mod Pathol 13: 577-585, 2000.

9. Miettinen $\mathrm{M}$ and Lasota J: Gastrointestinal stromal tumors: Pathology and prognosis at different sites. Semin Diagn Pathol 23: 70-83, 2006.

10. Terada T: Gastrointestinal stromal tumor of the uterus: A case report with genetic analyses of c-kit and PDGFRA genes. Int J Gynecol Pathol 28: 29-34, 2009.

11. Neto MR, Machuca TN, Pinho RV, Yuasa LD and Bleggi-Torres LF: Gastrointestinal stromal tumor: Report of two unusual cases. Virchows Arch 444: 594-596, 2004.

12. Yamaura K, Kato K, Miyazawa M, Haba Y, Muramatsu A, Miyata K and Koide N: Stromal tumor of the pancreas with expression of c-kit protein: Report of a case. J Gastroentero Hepatol 19: 467-470, 2004.

13. Krska Z, Pesková M, Povýsil C, Horejs J, Sedlácková E and Kudrnová Z: GIST of pancreas. Prague Med Rep 106: 201-208, 2005.

14. Daum O, Klecka J, Ferda J, Treska V, Vanecek T, Sima R, Mukensnabl P and Michal M: Gastrointestinal stromal tumor of the pancreas: Case report with documentation of KIT gene mutation. Virchows Arch 446: 470-472, 2005.

15. Showalter SL, Lloyd JM, Glassman DT and Berger AC: Extra-gastrointestinal stromal tumor of the pancreas: Case report and a review of the literature. Arch Surg 143: 305-308, 2008.

16. Yan BM, Pai RK and Van Dam J: Diagnosis of pancreatic gastrointestinal stromal tumor by EUS guided FNA. JOP 9: 192-196, 2008 .
17. Yang F, Jin C, Fu D and Ni Q: Extra-gastrointestinal stromal tumor of the pancreas: Clinical characteristics, diagnosis, treatment, and outcome. J Surg Oncol 103: 739-740, 2011.

18. Harindhanavudhi T, Tanawuttiwat T, Pyle J and Silva R Extra-gastrointestinal stromal tumor presenting as hemorrhagic pancreatic cyst diagnosed by EUS-FNA. JOP 10: 189-191, 2009.

19. Trifan A, Târcoveanu E, Danciu M, Hutanasu C, Cojocariu C and Stanciu C: Gastric heterotopic pancreas: An unusual case and review of the literature. J Gastrointestin Liver Dis 21: 209-212, 2012.

20. Goh BK, Kesavan SM and Wong WK: An unusual cause of a pancreatic head tumor. Gastroenterology 137: e5-e6, 2009.

21. Padhi S, Kongara R, Uppin SG, Uppin MS, Prayaga AK, Challa S, Nagari B and Regulagadda SA: Extragastrointestinal stromal tumor arising in the pancreas: A case report with a review of the literature. JOP 11: 244-248, 2010.

22. Saif MW, Hotchkiss S and Kaley K: Gastrointestinal stromal tumors of the pancreas. JOP 11: 405-406, 2010.

23. Rao RN, Vij M, Singla N and Kumar A: Malignant pancreatic extra-gastrointestinal stromal tumor diagnosed by ultrasound guided fine needle aspiration cytology. A case report with a review of the literature. JOP 12: 283-286, 2011.

24. Čečka F, Jon B, Ferko A, Šubrt Z, Nikolov DH and Tyčová V: Long-term survival of a patient after resection of a gastrointestinal stromal tumor arising from the pancreas. Hepatobiliary Pancreat Dis Int 10: 330-332, 2011

25. Vij M, Agrawal V and Pandey R: Malignant extra-gastrointestinal stromal tumor of the pancreas. A case report and review of literature. JOP 12: 200-204, 2011.

26. Soufi M, Bouziane M, Massrouri R and Chad B: Pancreatic GIST with pancreas divisum: A new entity. Int J Surg Case Rep 4: 68-71, 2013.

27. Kim HH, Koh YS, Park EK, Seoung JS, Hur YH, Kim JC Cho CK and Kim HJ: Primary extragastrointestinal stromal tumor arising in the pancreas: Report of a case. Surg Today 42: 386-390, 2012.

28. Beltrame V, Gruppo M, Pastorelli D, Pizzi S, Merigliano S and Sperti C: Extra-gastrointestinal stromal tumor of the pancreas: Case report and review of the literature. World J Surg Oncol 12: 105,2014

29. Hu X, Forster J and Damjanov I: Primary malignant gastrointestinal stromal tumor of the liver. Arch Pathol Lab Med 127: 1606-1608, 2003

30. Chen J, Du YJ, Song JT, ELN and Liu BR: Primary malignant liver mesenchymal tumor: A case report. World J Gastroenterol 16 : 5263-5266, 2010

31. Kim HO, Kim JE, Bae KS, Choi BH, Jeong CY and Lee JS: Imaging findings of primary malignant gastrointestinal stromal tumor of the liver. Jpn J Radiol 32: 365-370, 2014.

32. van der Zwan SM and DeMatteo RP: Gastrointestinal stromal tumor: 5 years later. Cancer 104: 1781-1788, 2005.

33. Shinomura Y, Kinoshita K, Tsutsui S and Hirota S: Pathophysiology, diagnosis, and treatment of gastrointestinal stromal tumors. J Gastroenterol 40: 775-780, 2005.

34. Rubin BP: Gastrointestinal stromal tumours: An update. Histopathology 48: 83-96, 2006.

35. Larsen LP: Role of contrast enhanced ultrasonography in the assessment of hepatic metastases: A review. World $\mathrm{J}$ Hepatol 2: 8-15, 2010.

36. Li R, Yuan MX, Ma KS, Li XW, Tang CL, Zhang XH, Guo DY and Yan XC: Detailed analysis of temporal features on contrast enhanced ultrasound may help differentiate intrahepatic cholangiocarcinoma from hepatocellular carcinoma in cirrhosis. PLoS One 9: e98612, 2014

37. Welch DR: Do we need to redefine a cancer metastasis and staging definitions? Breast Dis 26: 3-12, 2006.

38. Popescu LM, Hinescu ME, Ionescu N, Ciontea SM, Cretoiu D and Ardelean C: Interstitial cells of Cajal in pancreas. J Cell Mol Med 9: 169-190, 2005

39. Arora A and Scholar EM: Role of tyrosine kinase inhibitors in cancer therapy. J Pharmacol Exp Ther 315: 971-979, 2005. 